\title{
An integrated platform for smart energy management: The CC-SEM project
}

\author{
Una plataforma integrada para la gestión inteligente de la energía: El proyecto CC-SEM
}

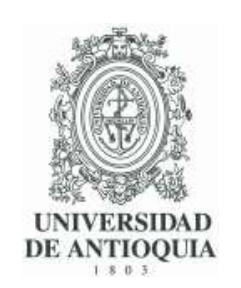

\author{
Emmanuel Luján (D ${ }^{1,4^{*}}$, Alejandro Otero (D) ${ }^{1,2}$, Sebastián Valenzuela ${ }^{3}$, Esteban Mocskos $\mathbb{D}^{1,4}$, Luiz Angelo Steffenel \\ (1) ${ }^{5}$, Sergio Nesmachnow (1) ${ }^{3}$ \\ ${ }^{1}$ Centro de Simulación Computacional para Aplicaciones Tecnológicas (CSC-CONICET). Polo Científico Tecnológico. Godoy Cruz 2390. \\ C1425FQD. Ciudad Autónoma de Buenos Aires. Argentina \\ ${ }^{2}$ Facultad de Ingeniería. Universidad de Buenos Aires. Av. Paseo Colón 850. C1063ACV. Ciudad Autónoma de Buenos Aires. Argentina \\ ${ }^{3}$ Universidad de la República. Julio Herrera y Reissig 565. 11.300. Montevideo. Uruguay \\ ${ }^{4}$ Departamento de Computación. Facultad de Ciencias Exactas y Naturales. Universidad de Buenos Aires. Pabellón I, Ciudad \\ Universitaria. C1428EGA. Ciudad Autónoma de Buenos Aires. Argentina. \\ ${ }^{5}$ Université de Reims-Champagne Ardenne. Moulin de la Housse. BP 1039. 51687 Reims CEDEX 2. France.
}

\begin{abstract}
CITE THIS ARTICLE AS:
E. Luján, A. Otero, S.

Valenzuela, E.

Mocskos, L. A.

Steffenel and S.

Nesmachnow. "An

integrated platform for

smart energy

management: The

CC-SEM project",

Revista Facultad de

Ingeniería Universidad

de Antioquia, no. 97.

pp. 41-55, Oct-Dec

2020. [Online].

Available:

https://www.doi.

org/10.17533/udea.

ABSTRACT: Energy management focuses on improving the efficient use of resources and increasing energy access in a path towards a more sustainable society. In spite of the strategies that have been proposed to guarantee increased access to the energy resources at affordable costs, there are still challenges to ensure the conservation of the resources and the protection of the environment. In line with these objectives, Cloud Computing for Smart Energy Management project (CC-SEM) is a research effort for building an integrated platform for smart monitoring, controlling, and planning energy consumption and generation in urban scenarios. CC-SEM includes, firstly, the design of a low-cost loT device capable of monitoring, operating, and controlling home appliances according to predefined rules. It was developed with the aim of automatically manage consumption. Secondly, an analysis of $5 \mathrm{G}$ Narrowband loT as a suitable cellular technology for Smart Grid outage restoration and management message delivery was addressed. Thirdly, an analysis of domestic consumption patterns was carried out to help to predict home consumption, using literature measurements. Fourthly, within the context of electrical network simulation, a forecasting and performance evaluation methodology for the generation of individual photovoltaic systems is proposed. In summary, CC-SEM presents the research efforts to provide a set of tools for controlling home devices, planning/simulating scenarios of energy generation, and to propose advances in the communication infrastructure for transmitting the generated data.
\end{abstract}

redin. 20191147

\section{ARTICLE INFO:}

Received: December

04,2018

Accepted: November

05, 2019

Available online:

November 05, 2019

\section{KEYWORDS:}

Smart cities; cloud computing; loT; 5G; energy efficiency

Ciudades inteligentes; computación en la nube; loT; 5G;

eficiencia energética

RESUMEN: La gestión energética se centra en mejorar el uso eficiente de los recursos y aumentar el acceso a la energía en camino hacia una sociedad más sostenible. En línea con estos objetivos, el proyecto Cloud Computing for Smart Energy Management (CC-SEM) investiga la construcción de una plataforma integrada para el monitoreo inteligente, el control y la planificación del consumo, y la generación de energía en escenarios urbanos. CC-SEM incluye, en primer lugar, el diseño de un dispositivo loT de bajo costo capaz de monitorear, operar y controlar electrodomésticos. Éste fue desarrollado con el objetivo de administrar automáticamente el consumo. En segundo lugar, un análisis de la idoneidad de la tecnología celular 5G NB-loT con respecto al envío de mensajes de restauración y gestión para interrupciones del suministro en redes eléctricas inteligentes. En tercer lugar, un análisis de patrones de consumo doméstico para ayudar a predecir el mismo, utilizando mediciones de la literatura. En cuarto lugar, dentro del contexto de simulaciones de redes eléctricas, una metodología de pronóstico y evaluación de rendimiento para la generación de sistemas fotovoltaicos. CC-SEM presenta avances respecto del control de dispositivos domésticos, planificación/simulación de escenarios de generación de energía, y propone avances en la infraestructura de comunicación de los datos generados.

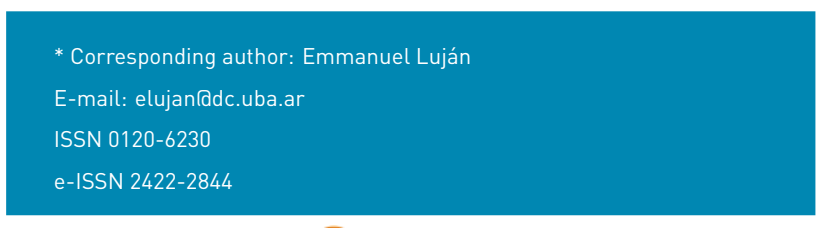




\section{Introduction}

Energy management is a crucial issue in modern society. Many strategies have been proposed to guarantee increased access to the energy resources at affordable costs for citizens while ensuring the conservation of the resources and the protection of the environment [1].

For the implementation of effective energy management policies, innovative technologies must be integrated into an easy-to-use and efficient system to include the capabilities of performing realistic simulations, controlling and planning the electricity market (to be applied by the energy companies), and end-user applications to monitor and manage the energy consumption at home level. The capabilities of monitoring/controlling/managing the energy consumption and generation are key issues when implementing the smart city paradigm, especially when considering the emphasis on citizen engagement, environment protection, and economic considerations [2].

This article describes the Cloud Computing for Smart Energy Management (CC-SEM) project, developed by researchers from Argentina (Consejo Nacional de Investigaciones Científicas y Tecnológicas (CONICET) and Universidad de Buenos Aires (UBA)), Uruguay (Universidad de la República (UdelaR)) and France (Université de Reims-Champagne Ardenne (Reims)), and presents preliminary results. CC-SEM proposes developing an integrated platform for smart monitoring and controlling the energy consumption in urban scenarios, by integrating Big Data analysis, computational intelligence, Internet of Things (loT), High Performance, and Cloud Computing. In our Latinamerican region, there have been some limited developments towards building some specific components for energy management, but no global solutions have been explored or made available to the public. As a consequence, the CC-SEM project proposes a useful system with real application and social relevance.

Nowadays, integrating renewable energy is a relevant interest nowadays, as part of a global effort to reduce the effect of the $\mathrm{CO}_{2}$ emissions [3, 4]. However, this integration poses a big challenge for the operation of the energy grid, due to the unpredictable nature of some of the renewable energy sources, such as wind and solar. Instability on renewable energy affects the electric grid, causing voltage fluctuations, changes on current and frequency, etc. In this scenario, the utilization of specific techniques for smart grid management is mandatory. Conceiving an automatic management strategy that works correctly on macro scenarios lenergy grid management and energy distribution) and micro scenarios (guaranteeing the appropriate quality of service for users) is not an easy task. One viable alternative is using mathematical models and computational intelligence techniques for planning and operating the energy distribution and utilization in real-time. To apply intelligent management systems, specific hardware is needed to evaluate and control energy consumption by using sensors, data communications, and control devices. These devices must be able to communicate between them and with central servers to integrate all the logic of the system and determine quick responses to different dynamic situations /sudden increase in energy consumption, reduction in energy generation, increase on the energy generation costs). Recently, new smart consumption monitors were made available to be installed. For example, the Linky smart meter [5] by Électricité de France (EDF) that is being widely deployed, allowing not the collection of consumption but also some remote actions. Other projects such as ElectriSense [6] can even identify the families of devices consuming energy. None of these systems are open-source and their capabilities are often restricted to data acquisition and basic automation.

Having sensors to generate data consumption measures is not enough. Strategies to transmit and use this information should be developed and adopted by users and companies. Understanding and applying computational intelligence algorithms is one of the possible paths to analyze this data, determining routines and patterns of energy utilization by individual users. Another option is planning strategies to optimize the energy consumption, by deciding when to power on and off each device from the home, building or neighborhood. The planning strategies will have into account the user restrictions and support real-time actions from the user without having a critical impact on the planning. According to the capabilities of the devices, this planning can be fully automatized using IoT actuators or manually, by suggesting actions to the user via the smartphone interface [7-9].

In the context of microgrids, other projects have been proposed. Particularly, in [10], a smart energy management system (SEMS) was presented, addressing some of the matters exposed above. SEMS optimally coordinate the power production of distributed generation sources and the energy storage system, minimizing the operational costs.

CC-SEM project addresses the aforementioned issues, by proposing a research effort focused on building an integrated platform for smart monitoring, controlling, and planning energy consumption and generation in urban scenarios. This article is an extended version of our conference article [11]. The main contributions of the scientific content reported in this article include: i) a detailed description of the project goals and activities, ii) an extended analysis of the obtained results, including a 
description of the low cost energy consumption monitor and controller, extended results on the characterization of domestic energy consumption and the comparison of two process pipelines for the forecast of urban photovoltaic energy generation, and iii) an analysis of the suitability of NB-IoT as the smart meter communication technology for the proposed platform.

The article is organized as follows. Section 2 presents an overall description of the project. The main activities within the project are described in Section 3. Preliminary results are reported in Section 4. Finally, some conclusions about the ongoing work and the main lines for future work are formulated in Section 5.

\section{Project description}

This section describes the main features of the CC-SEM project.

\subsection{Project goals and motivation}

The main goal of the project is to design a platform that allows the integration of fundamental concepts and tools for energy management in smart cities, using cloud computing, computational intelligence for big data analysis, and software for simulation and optimization of the energy generation and distribution. The aim is providing both users and administrators of the electrical grid a useful set of tools for intelligent planning and organization of the electricity consumption and generation nowadays and in future smart cities. From the point of view of the users, the project proposes the design and management of a smart home controller for electric devices applying loT related software, and the application of Big Data processing techniques for the analysis of domestic energy consumption and smart planning. From the point of view of the electric grid administrators, novel tools are presented to monitor the state of the network and the overall quality of services, and the use of novel simulation tools is proposed to analyze and foresee the energy demand. This approach is planned to be adopted by electric market regulators in Argentina and Uruguay.

Energy optimization and planning is on the agenda of many countries. Only few solutions implement an easy-to-use platform to be used by both end-users and energy companies integrating hardware, software, and communications. Energy providers have few (or even no) knowledge about electricity utilization in homes. With the current electrical infrastructure, the provider cannot determine if the energy is well-used or wasted. Having a hardware infrastructure that allows obtaining useful information about utilization is the first stage in a global system to optimize energy at homes, with the main goals of reducing the costs of energy consumption and generation, and improve the quality of service offered to the users.

Another important motivation of the project is to conceive a generic set of tools to allow both users and administrators to extract useful information from the raw data measured by the home controller by applying computational intelligence/machine learning techniques. We propose basing the communications on the IoT paradigm to guarantee ubiquitous access to the system, everywhere, anytime, and using a wide range of communication devices (smartphones, tablets, web interface, other management systems, etc.). The applications that integrate the proposed system need to be conceived as a part of a global monitoring/planning system to be used in real-time in modern smart cities.

\subsection{Methodology}

The proposed methodology, in line with the project goals, is two-fold. On one hand, the methodology is based on missions that help the project members to consolidate a collaboration network. The institutions have been in contact in the past: research groups at UdelaR and Reims have collaborated in research activities related to distributed computing and cloud computing, and research groups at UdelaR and UBA have collaborated in research activities related to high performance/distributed computing and applications. However, CC-SEM is the first initiative to set a common project between the three institutions. On the other hand, the research subject is realistic and represents a real need, as observed in recent contacts with Academia, Industry, and social actors related to energy management and the real implementation of the smart cities paradigm.

Due to the interdisciplinary nature of the project, and the fact that several actors (e.g., users, companies, agencies) are interested in the project outcomes, all results achieved during the project are being rapidly made available to the community via the project website, public repositories, and on seminaries, meetings, and conferences.

\section{Project activities}

This section describes the main project activities, which are related to the contributions reported in this article. In Figure 1, an abstract representation of the proposed system is presented. Generators and households, from generation and distribution stages, respectively, are monitored and controlled by dedicated devices connected to a management system lusing cellular or wired networks). In line with this view, current research efforts were concentrated on three major axes: 
1. Automatic energy management for home devices. This axis aims at designing a hardware platform for monitoring and controlling domestic consumption, following an open architecture approach, to allow future expansions. The loT paradigm is applied to support communication between components, and guarantee ubiquitous access to the proposed controller and software tools to be developed within the project. In this regard, a specific low-cost energy consumption monitor and controller was developed, whose main details are presented in Section 4.1.

2. Big Data analytic for domestic energy consumption and smart planning. This axis refers to extracting useful information to be considered when planning the energy utilization (by end-users) and generation (by energy companies). It focuses on developing integrated methodologies and techniques for extracting useful information from the raw energy consumption data, to be used in smart energy management and optimization in urban scenarios, regarding energy utilization, estimation of the economic cost, and maintaining a good quality of service for users. Section 4.3 presents the application of computational intelligence techniques for the characterization of household energy consumption.

3. Tools and algorithms for electrical network simulation. One defining characteristic of smart grids is that both power and information flow in both senses, from and to the consumers, who will take a more active role. The goal of this axis is to build a unified computational framework to simulate smart grids with the capability of analyzing a national-wide power network under typical situations of interest for the different actors of the power sector: generators, carriers, utilities, consumers, planners, developers, decision-makers, etc. In this context, with an increasing number of devices, effective data communication strategies are needed. Some devices can communicate directly to the home user gateway and the Internet, but many of them cannot [12]. In this regard, Section 4.2 presents a narrow-band protocol for smart grids and its evaluation over different urban scenarios, and Section 4.4 describes the application of computational intelligence techniques for renewable energy generation forecast.

\section{Initial advances}

This section reports partial results obtained in the context of the CCSEM project.

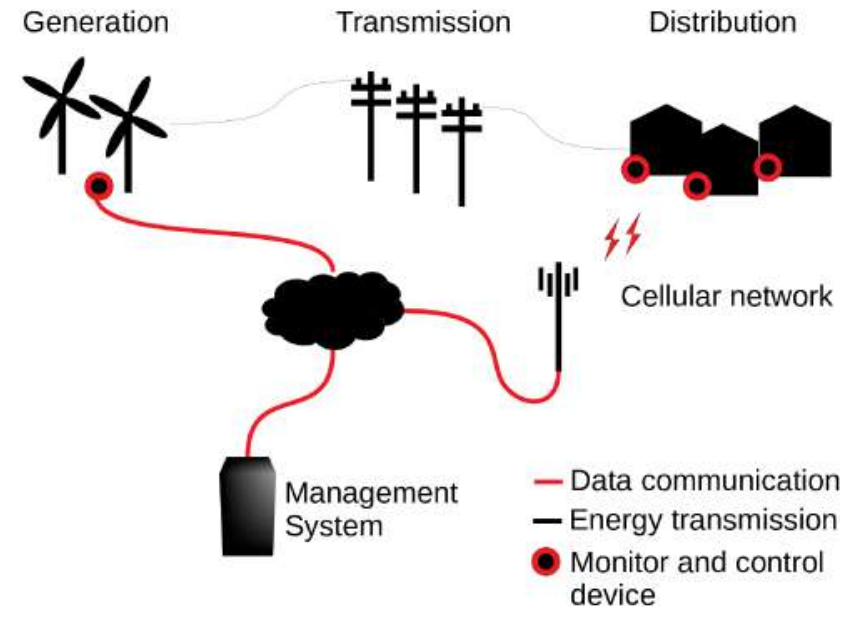

Figure 1 Electrical network main stages and its communication with the management system through the monitor and control devices

\subsection{Low cost energy consumption monitor and controller}

A prototype for the smart metering system was designed and built according to the general specification from our project. The proposed system integrates three components: i) a specific module and protocol (Energy Efficiency, EFEN), which allows defining/storing user actions and preferences, and compute plannings; ii) the monitor/controller itself, and iii) an interface for communication with the home controllers, based on Khimo framework. These components are implemented in independent modules that allow monitoring, operating, and controlling home devices according to specific rules.

EFEN defines home devices, stores power consumption data, provides an interface for device control, and also computes ad-hoc planning taking into account user preferences. In EFEN, electric devices are grouped in homes, but larger aggregations are also supported: homes can be grouped in buildings and buildings can be grouped in neighborhoods. This categorization allows performing energy planning at different levels, according to the preferences of single users/community users, and/or the needs of electric companies. EFEN also provides an Application Programming Interface (API) meant to implement the integration of computational intelligence algorithms for big data analysis/pattern recognition and energy planning, to be designed in WP 4. EFEN also includes a feasibility check for defined agendas, a tool to simulate historical power consumption time series (useful for verification purposes), energy and cost evaluation, and user satisfaction estimation algorithms.

The controller, presented in Figure 2, is based on a 


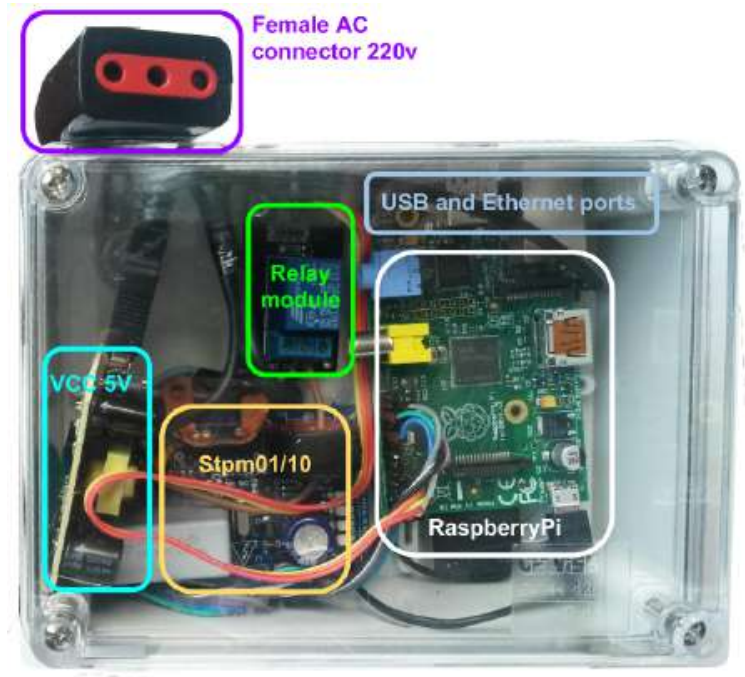

Figure 2 Prototype of the low cost energy consumption monitor and controller with detailed components

Single Board Computer (Raspberry $\mathrm{Pi}$, providing a flexible and portable solution) that controls other two modules: i) a power meter STPM01/10, integrated using the steval-ipe016v1 board, which performs the measurements using the SPI protocol, and ii) a relay for power supply control. The controller communicates with the central system (Khimo) via the Internet. The system is controlled by the Khimo module, which allows performing communications via the loT paradigm [13] to guarantee ubiquitous access (everywhere, anytime), and using multiple communication devices (e.g., smartphones, tablets, web interfaces, etc.). Khimo allows remote monitoring and controlling of several devices in real-time.

A specific protocol (EFEN_PROTO) was developed to guarantee efficiency in the communications between Khimo and EFEN, and also to enable controlling devices. Using a bidirectional communication channel, enabling event subscription, and avoiding intermittent queries (i.e., polling) to each controlled device, EFEN_PROTO provides efficiency for gathering power consumption and other information from devices, and also to define actions according to the state of home appliances. Action grouping is applied to deliver messages of the same type. By grouping home appliances according to the actions to perform over them, EFEN_PROTO avoids redundancy, thus improving the communication efficiency.

A greedy algorithm was proposed as a first step to design computational intelligence methods for home energy planning. This greedy algorithm focuses on minimizing power consumption and maximizing user satisfaction, by taking local decisions to build a global agenda and considering the maximum power available is a hard restriction.
The main details about the design of the controller were published in [14] and the application of loT-based information for designing simple heuristics for smart home energy planning is described in [15]. Further details can be found on the website https://www.fing.edu . uy/inco/grupos/cecal/hpc/EFEHO.

\subsection{NB-IoT suitability for smart grid metering}

To assess the state of smart grids, smart meters are deployed to monitor the grid. Those devices inform magnitudes that can be used to derive information about the whole urban scenario. Thus, the supporting communication network plays a fundamental role to ensure collecting state information. While wired networks entail high economic costs, wireless networks are positioned as a competitive alternative [16]. In particular, the exponential growth of cellular wireless networks establishes a powerful infrastructure for new communication technologies. In recent years, a narrow-band radio technology (less than $200 \mathrm{kHz}$ ) has been developed: Narrow Band Internet of the Things (NB-IoT) [17]. It is designed to satisfy requirements of low-bitrate applications, with special emphasis in coverage enhancement, ultra-low power consumption, and massive terminal access.

Another characteristic of this technology is non-latency-sensitivity, despite this, high channel occupation scenarios occur, thus, increasing latency levels over tolerable thresholds. In particular, when a local or regional blackout occurs, a massive number of Outage Restoration and Management (ORM) messages are sent from the smart meters at the households to the energy provider company. Each message has a payload of 200 bit and a maximum latency of $20 \mathrm{~s}$. Latter issue is critical for a Smart Grid platform, ergo any metering communication technology should handle it appropriately. In [18], an assessment of this problem was carried out. Here we extend previous analysis proposing two strategies for handling massive simultaneous ORM messages, guaranteeing that all of these messages will arrive correctly:

- To provide communication to a smaller number of smart meters decreasing the base station (BS) footprint.

- To utilize machine-to-machine (M2M) communication for aggregating ORM messages between the smart meters.

Our analysis assumes the following pessimistic scenario: a low signal-to-noise (SNR) ratio of $-20 \mathrm{~dB}$, just one sub-carrier available for channel usage (single tone), frequency numerology of $3.75 \mathrm{kHz}$ which sets resource 
unit (RU) time to $32 \mathrm{~ms}$, and re-transmissions are not available (unacknowledged mode).

By taking into account the uplink sub-process in which the smart meter sends a transfer block of 200 bit to the BS we establish a maximum number of RUs that can be transmitted during the first $320 \mathrm{~s}$ after the failure occurred in Equation 1.

$$
\mathrm{RUs}_{\text {max }}=\frac{\text { Response }_{\text {time }}}{R U_{\text {time }}}=\frac{320 \mathrm{~s}}{32 \mathrm{~ms}}=10^{4}
$$

Assuming each household (HH) has a single smart meter, the maximum number of households that can transmit its ORM message during the latency interval is presented in Equation 2.

$$
\mathrm{HHs}_{\max }=\frac{R U s_{\max }}{R U s_{O R M}}=\frac{10^{4}}{1024} \cong 10
$$

RUs $O R M$ is the number of RUs needed for an ORM message in the established pessimistic scenario. To obtain this value, we developed a lite NB-IoT simulator for the uplink layer based on the Matlab Toolkit module: Uplink Waveform Generation. The link-layer model is implemented according to the NB-IoT physical uplink shared channel (NPUSCH) standard $([19,20])$. Montecarlo simulations are performed over an AWGN channel to obtain block error rate (BLER) information for the different link-layer configurations. The outcome of the simulation is used to trace BLER curves and obtain optimal link settings for target BLERs. This software was previously used [11, 21] to asses strategies for decreasing radio resource consumption. A detailed description of the current version is also presented in [22]. The whole project source code can be found in [23]. Table 1 has an extract of the simulation results.

Table 1 Extract of table generated by our NB-IoT uplink simulator. Table columns inform the transfer block size, effective SNR, target BLER and the amount of RUs needed to transfer the information

\begin{tabular}{|c|c|c|c|}
\hline TBS $\backslash$ bits & $S N R \backslash \mathrm{dB}$ & BLER & $R U s$ \\
\hline \hline$\ldots$ & $\ldots$ & $\ldots$ & $\ldots$ \\
\hline $\mathbf{2 0 8}$ & $\mathbf{- 2 0}$ & $\mathbf{0}$ & $\mathbf{1 0 2 4}$ \\
\hline 208 & -20 & 0.0037 & 192 \\
\hline 208 & -20 & 0.1518 & 160 \\
\hline$\ldots$ & $\ldots$ & $\ldots$ & $\ldots$ \\
\hline
\end{tabular}

Table 2 present household densities $\left(\rho_{H H}\right)$ for different urban scenarios [18]. In NB-IoT, coverage radio is less than $15 \mathrm{~km}$, i.e a maximum footprint area of $706 \mathrm{~km}^{2}$. To guarantee that $H H_{\max }$ smart devices can send its ORM messages concurrently, the footprint can be decreased. Last column of Table 2 shows footprint radio $\left(\mathrm{Fp}_{r}\right)$ for the different environments. The weakness of this strategy is that the number of BSs that should be installed to provide coverage to the original area significantly increases.

Table 2 NB-IoT base station footprint vs household density for aggregation factor $\alpha=1$

\begin{tabular}{|c|c|c|}
\hline Environment & $\rho_{H H} \mid \mathrm{HHs}_{\mathrm{km}} \mathrm{Km}^{-2}$ & $F p_{r} \mid \mathrm{m}$ \\
\hline \hline Dense Urban (DU) & 2272 & 37 \\
\hline Urban (U) & 1500 & 46 \\
\hline Suburban (SU) & 350 & 95 \\
\hline Rural (RU) & 50 & 252 \\
\hline
\end{tabular}

Utilizing M2M communication can mitigate latter issue. This strategy is based on $(\alpha-1)$ households sending their information, i.e. ORM messages, to a common neighbor, which in turn process them to generate an aggregated message, that is sent to the common destination.

In this analysis, we suppose that the message does not increase its size with respect to the original ORM message. On the other hand, the consensus time, i.e. the interval in which the smart meters send its messages to its neighbor and it performs the processing for aggregating those messages, is negligible. Both tasks take only a few milliseconds (see [24]), which is orders of magnitudes smaller than the maximum latency of the ORM message.

Equation 3 presents the function that determines BS footprint radio with respect to household density and the aggregation factor $\alpha$.

$$
\begin{gathered}
\rho_{H H, \alpha}=\frac{\rho_{H H}}{\alpha} \\
\pi F p_{r}^{2}=\frac{H H s_{\max }}{\rho_{H H, \alpha}} \\
F p_{r}\left(\rho_{H H}, \alpha\right)=10^{3} \sqrt[2]{\frac{\alpha H H s_{\max }}{\pi \rho_{H H}}}
\end{gathered}
$$

Figure 3 and 4 were generated based on the Equation 3, and present different views of the magnitudes involved on it. As can be observed, the augmentation of household density decrease NB-IoT base station footprint radio, but it can be compensated with the M2M aggregation factor $\alpha$. Particularly, in Figure 3 densest environment, footprint radio reaches $37 \mathrm{~m}$ when no $\mathrm{M} 2 \mathrm{M}$ aggregation is used, and rise up to $299 \mathrm{~m}$ aggregating 64 devices.

When is not necessary to send $H H_{\max }$ ORM messages concurrently, M2M aggregation can be used to reduce radio resource usage within the maximum latency interval. Therefore, this could increment the number of available RUs which can be used for other user equipment 


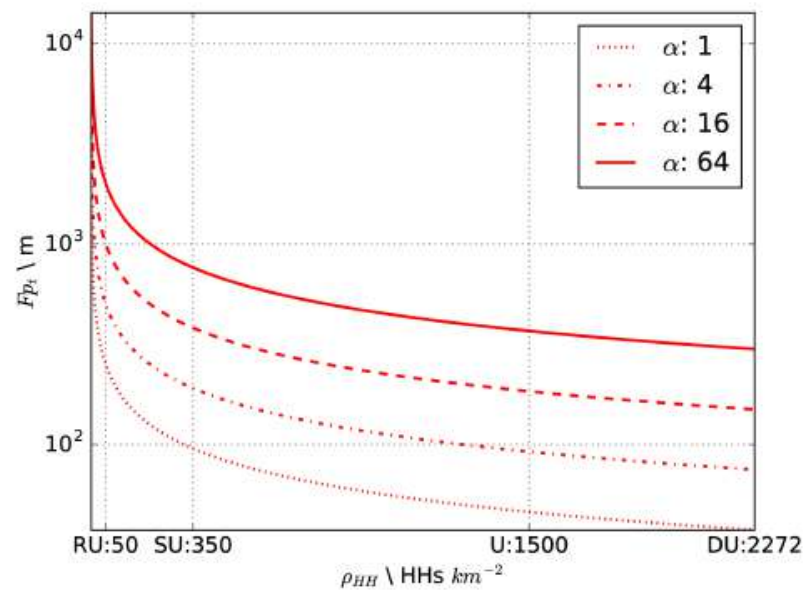

Figure 3 NB-IoT base station footprint vs household density for different aggregation factors $(\alpha)$

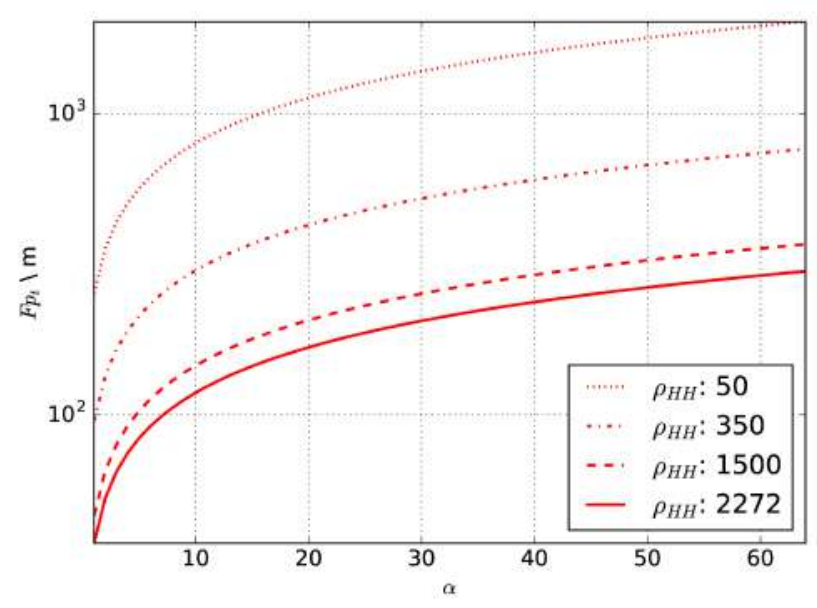

Figure 4 NB-IoT base station footprint vs aggregation factors $(\alpha)$ for different household densities $\left(\rho_{H H}\right)$

applications. Equation 4 defines the amount of available resource units depending on the transmission conditions:

$$
A=\sum_{i=0}^{N-1} R U s\left(T B S_{i}, S N R_{i}, B L E R_{i}\right)
$$

where TBS is the transfer block size, SNR is the signal-to-noise ratio and BLER is the block error rate. The upper bound of the magnitude presented in Equation 4 can be calculated, it is presented in inequality (5). Figure 5 shows how the upper limit of the available radio resources within the maximum latency interval varies for different number of OMRs and for different M2M aggregation factors.

$$
A<R U s_{\max }-O R M s R U s_{O R M}
$$

In conclusion, we address a critical communication metering issue in a Smart Grid platform: to successfully

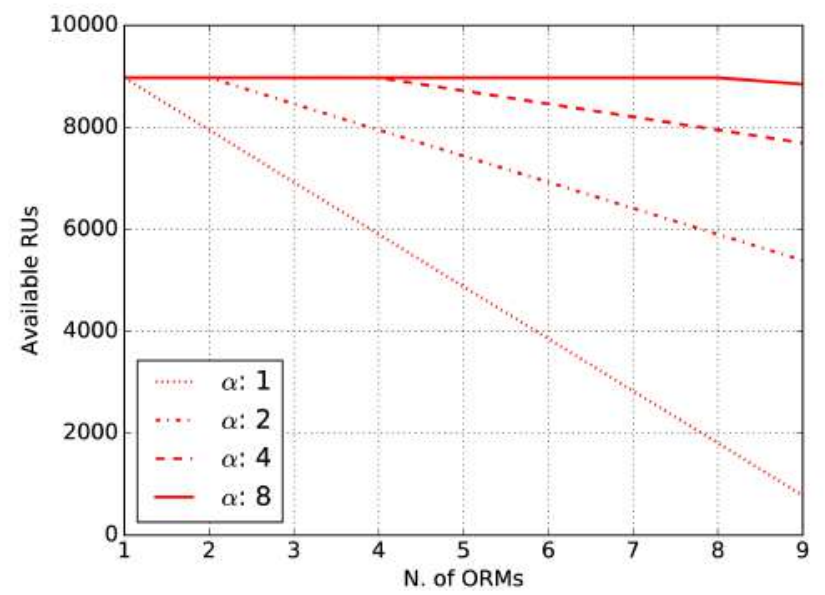

Figure 5 Available RUs during the response time vs the number of ORMs for different aggregation factors $(\alpha)$

deliver massive concurrent ORM messages according to latency constraints. An assessment of NB-IoT suitability as a communication technology for this matter was performed. We proposed two strategies for adapting NB-IoT capabilities to this latency problem: BS footprint adjustment and M2M aggregation. Our results suggest that NB-IoT is a suitable technology for smart metering over a Smart Grid context, in particular for the platform that is proposed in this work.

\subsection{Characterization of domestic energy consumption}

In the previous section, we presented our efforts to measure and collect data from a consumer, helping her to identify usage patterns and situations that can potentially reduce the energy consumption. Even though, the domestic energy consumption is not only a consumer problem but also affects the infrastructure on building and neighborhoods, especially in the case of old and overloaded power grid networks. With the massive expansion of smart meters, the opportunity to gather costumers data and use big data algorithms open the door to extract useful information at different levels, from the individual user to the entire grid itself.

In our project, one of the goals is to identify potential peaks that, combined with the consumption from other users, may lead to disturbances in the local distribution grid lin a building or the neighborhood). Hence, we try to predict situations that may stress the grid and trigger measures to avoid the overload, be them passive (warning the users) or active (automatic shutdown of devices). This is indeed a problem that affects some power networks such as those from Buenos Aires, where the fast development of residential areas was not followed by infrastructure 


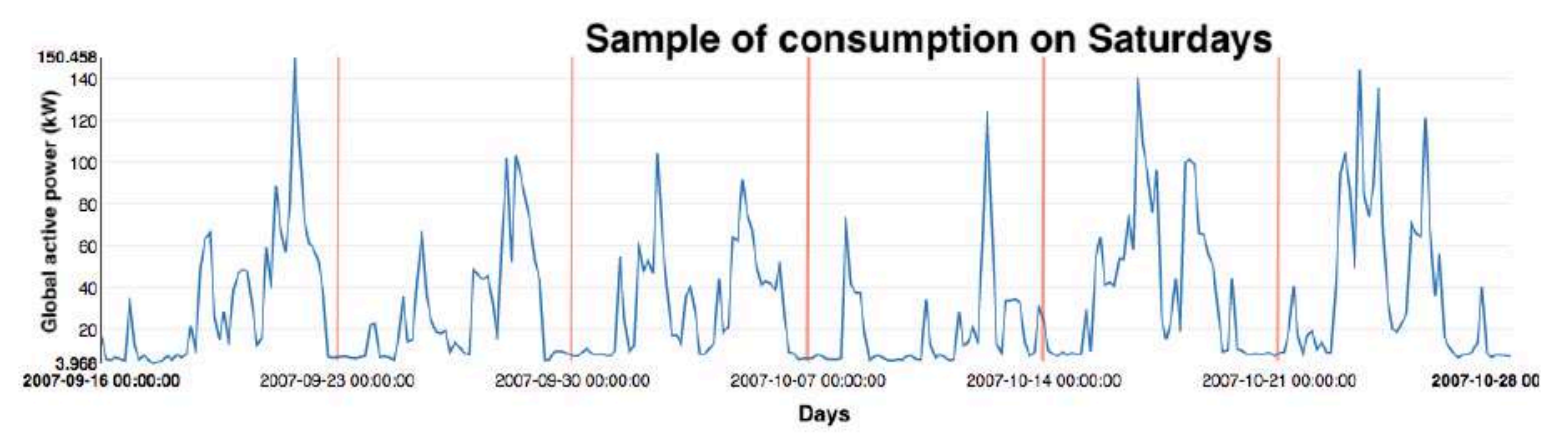

(a)

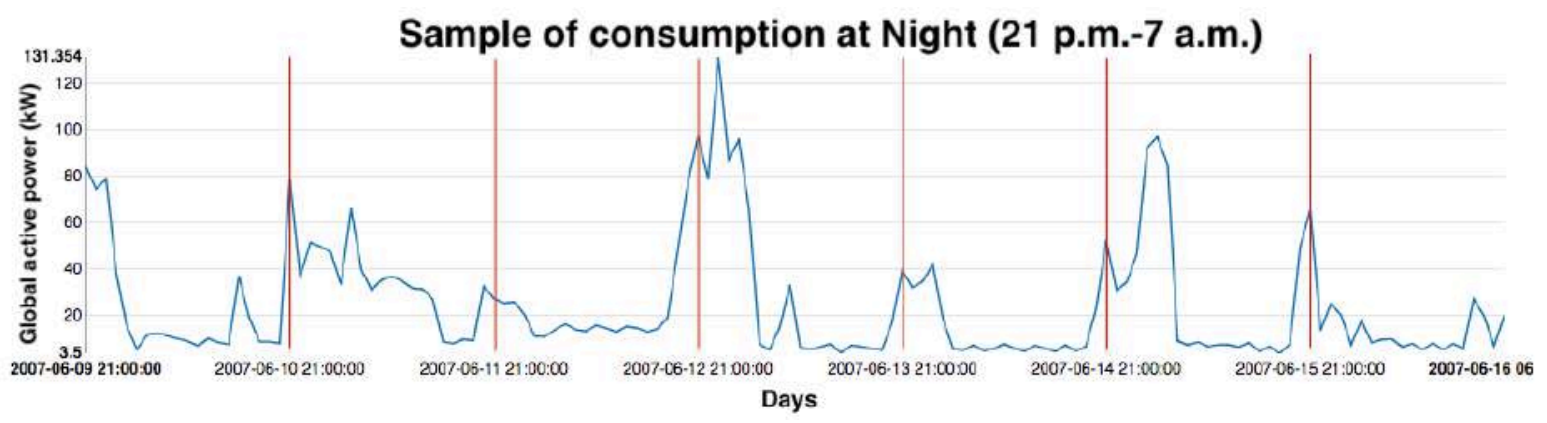

(b)

Figure 6 Example of individual consumption classified by day of the week (a) or by time of the day (b)

investments. Some projects like "Que no se corte" [25] try to handle this problem voluntarily: through a phone application, users sign in and feed basic information such as their location and electrical devices. Later, the "Que no se corte" application would send messages to encourage users to change their usage patterns.

Crowdsourcing efforts such as "Que no se corte" are necessary but they often struggle with the limited information they can explore. Indeed, having access to information on the power infrastructure is not always easy (for example, the location and capacity of the electrical transformers). Also, the usage profile differs from one user to the other. For instance, in the literature we mostly found methodologies to estimate the electric load at the system level. As illustrated by [26, 27], the electrical consumption at the system scale often follows seasonal variations at macro and micro scale (seasons, weekdays, an hour of the dayl, and the aggregation of several customers profile produces a smooth profile with consistent patterns that favor the forecasting accuracy. Unlike the system-level load, the individual residential consumption depends on the daily routine and users lifestyle but also on other elements that are harder to predict. While it is easy to forecast the consumption of a programmable water heater, it is much harder to estimate the consumption of other devices that may be activated alone or at the same time (Does one always turn on the oven and the dishwasher at the same time?). Some external elements such as the air temperature and the external weather can help to improve the predictions, but they also have a limited impact and correlation, depending on the residential characteristics (solar exposure, gas or electric heating), the thermal inertia of the buildings and the psychological resiliency of the inhabitants.

Several datasets for energy consumption can be found in the literature le.g., a complete list is presented on http://wiki.nilm.eu/datasets.html). We start analyzing the Individual household electric power consumption dataset [28] as this dataset covers more than three years of consumption of a house located at the south of Paris, France. It presents the overall consumption and the detail of specific sets of devices, with a resolution of one minute between measures.

Our first analysis involved the attempt to extract consumption patterns, like for example the profile for each weekday or for different periods of the day (dawn, morning, afternoon, night), which are reputed to present similar behaviors. Neither of the categories we tested led to conclusive results, as illustrated in Figure 6. Indeed, we see that two different days/periods have different profiles even if they belong to the same categories, making it hard to forecast the residential consumption. We also tried to correlate the consumption with the local weather, but 
the residence from this dataset seems to rely on other energy sources for heating and cooking (gas or oil), so the electrical consumption profile was driven mostly by less powerful devices that have a strong dependence on the user's habits. While a few patterns pointed in [29] could be extracted, they have small importance in the overall consumption and do not help to forecast.

As specific seasonal patterns could not help the prediction of the residential consumption, our next approach was to use deep learning techniques such as Long-Short Term Memory (LSTM). LSTM is a type of recurrent neural network designed for sequence problems such as time-series analysis and forecast.

In our specific case, we aimed at training the model to predict the consumption of the residence based on a sequence of previous measurements. This is not the first time LSTM has been used to this purpose [26, 27, 30], comforting our choice. However, the difference is that we are not interested in developing specific models for the forecast. Instead, we focus on the accuracy of simple models that could be later integrated into smart-meters with low CPU requirements. Because of this choice, we implemented a simple LSTM network using the Keras library, training it with the same dataset from Hebrail and Berard. After a few experiments, we chose a pipeline composed of one LSTM layer and two dense layers, as depicted in Figure 7.

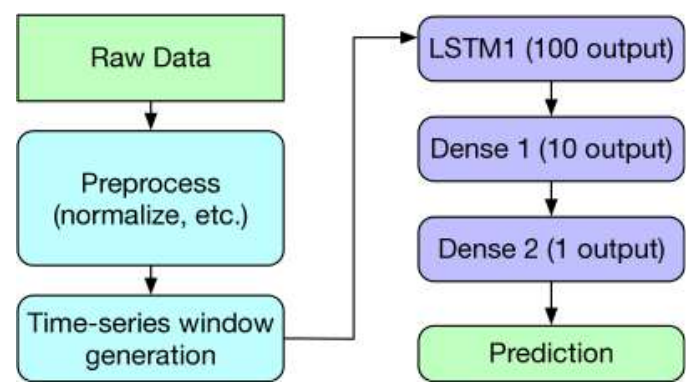

Figure 7 Structure of the forecasting pipeline

Hence, the network has a visible layer with one or several inputs depending on the length of the historical window we want to evaluate (for example, a $24 \mathrm{hs}$ windows with). It also includes a hidden layer with 100 LSTM blocks or neurons, a hidden dense layer with an output size of ten and a final dense layer that makes a single value prediction. The default sigmoid activation function is used for the LSTM block, and the last layer has a non-negative activation constraint (https://keras.io/constraints/) to prevent the algorithms to output negative values, which is unrealistic in the case of power consumption. The "inner" dense layer is not required but seems to produce smoother predictions.
The training was made with $30 \%$ of the dataset (what roughly corresponds to a year of measures). The network was trained for 150 epochs and a batch size of 1 . The resulting trained model was able to give good predictions on the remainder of the dataset (RMSE=22), as illustrated in Figure 8.

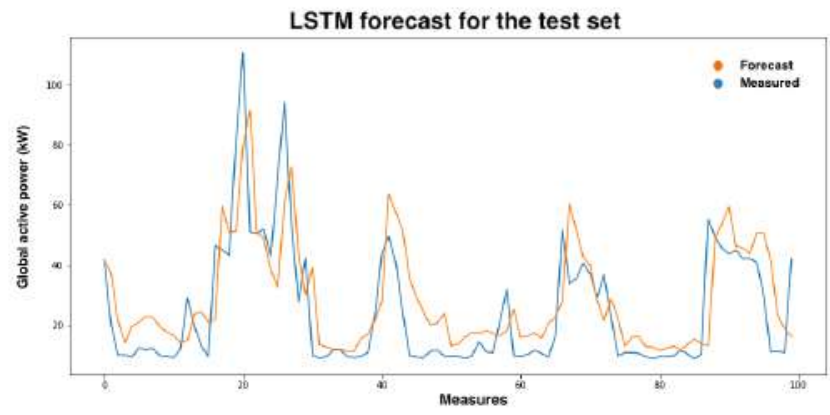

Figure 8 Sample of LSTM forecast for the reference dataset

One inconvenient of applying deep learning techniques to the consumption of each residential user is that we need sufficient data to train the model. Indeed, good training requires at least a few months of readings, which would delay the start of operations for new costumers. As a consequence, we decide to circumvent this drawback by applying an existing model (from another residence) and verifying its effectiveness. For this reason, we do not try to optimize the parameters or develop more elaborated LSTM networks, but instead, we focus on applying the trained LSTM model over an independent dataset lavailable at http://cosy.univ-reims.fr/ lsteffenel/documents/residential.zipl, obtained from a real user thanks to the Linky smart meter [5].

Contrarily to the reference dataset, this residence fully relies on electricity for heating and cooking, which can raise different consumption profiles. Three different intervals were compared as input history for the LSTM model: one day, one week and four weeks (roughly a month), as illustrated in Figure 9. The analysis of these forecasts shows that using only $24 \mathrm{hs}$ of history brings insufficient input data, and the forecast tends towards the "persistence" of the previous state. Forecasts with a month of history are better but, on the contrary, tend to smooth the consumption and raise the expectation for the lower values. Finally, the forecasts made with a week of history seem to offer a better trade-off between accuracy and the history length.

While prediction errors still occur (mostly "false positive" forecasts, like for example on the mark 1-20 on the samples), these estimations are good enough to help a recommendation system or to help to detect potential 


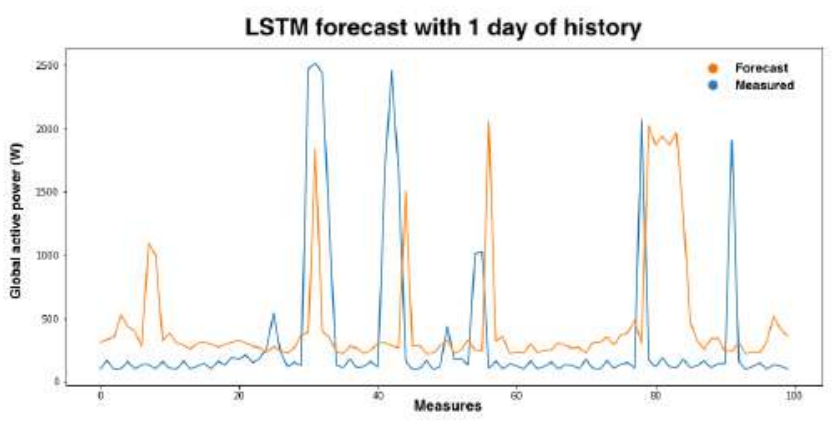

(a)

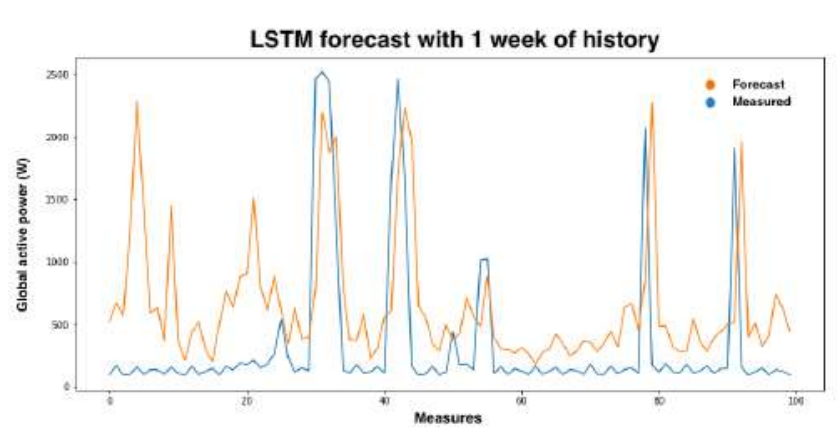

(b)

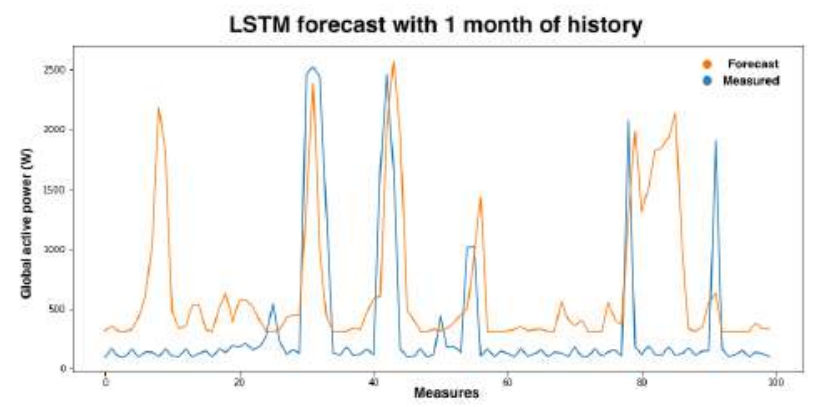

Figure 9 LSTM forecasts with different history lengths: (a) 1 day, (b) 1 week and (c) 1 month

overloads (summing up the expected consumption from several residences). As expected, the RMSE is still important (about 300) but at least we have a baseline model that can be quickly deployed. As soon as more measurements are collected, individual models can be created by incrementally training the model. Also, specific parameter optimizations can be performed to improve the accuracy.

The scalability and portability of the developed solution can be ensured by relying on basic ETL (Extraction, Transformation, Load) using MapReduce, while more advanced operations can be conducted with high-level tools (e.g., Pig or Apache Spark and Tensorflow/Keras). Storage and access to the data can be made using NoSQL databases, which are specially adapted to store
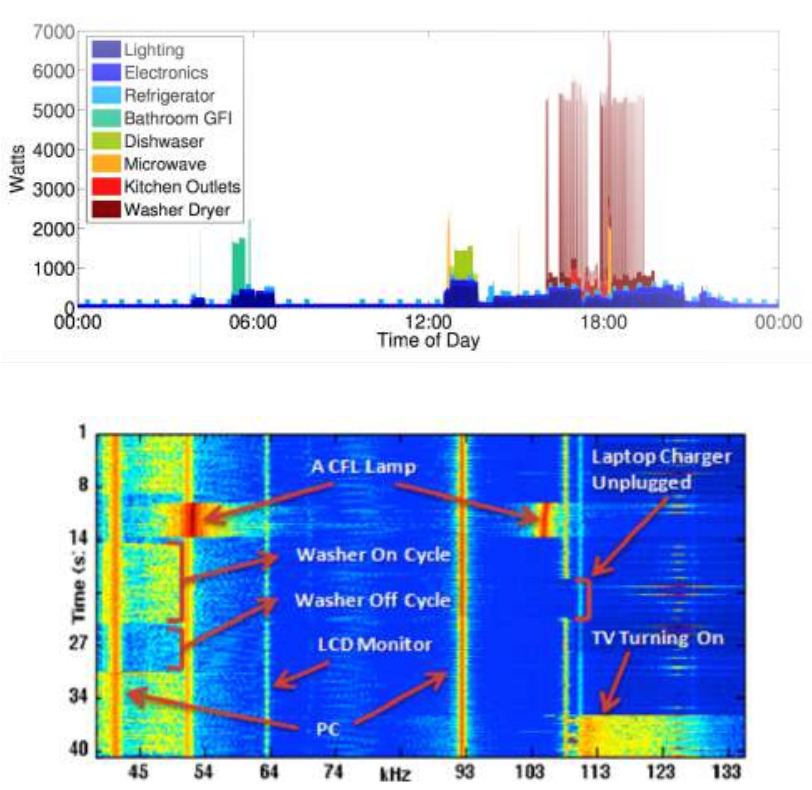

Figure 10 Different examples of non-intrusive load monitoring (NILM) $[6,32]$

data series from sensors and other data sources le.g., power generation, historical consumption charts, weather forecast) [31].

Further developments can include energy disaggregation, also referred to as non-intrusive load monitoring (NILM). NILM is a technique to make inferences about the different individual loads of the system based on an aggregate energy signal, such as that coming from a whole-home

(c) power monitor. NILM is a full research domain with several challenges, such for example the identification of devices signatures even when they do not work always in a constant regime (Figure 10). Indeed, most devices present acyclic consumption behaviors, like for example the different phases of a washing machine program. An extended library of NILM datasets and papers can be found at http://wiki.nilm.eu/datasets.html.

In the case of our project, we can focus on the identification of power-hungry devices, like heaters and air conditioning systems. Compared to other devices like TVs, lamps or refrigerators, these devices may induce peaks of consumption that may overload the distribution system. By detecting their activity we can send warnings to the users (using the "Que no se corte" application, for example) or even trigger controlled shutdowns thanks to smart power outlets connected to the metering system. 


\subsection{Renewable energy generation forecast}

Although there are some options to produce energy from wind and other sources, photovoltaic (PV) systems are the most likely way to generate centralized or distributed renewable energy in an urban setting in a massive scale. The challenges for adopting higher shares of this type of energy are posed by its intermittence, inherited from solar radiation dependency on local weather, mainly the cloud cover and optical thickness. Aiming to forecast PV generation for a particular system in a specific location, both the resource and the system characteristics should be considered. The capacity of forecasting PV generation for different installations will help manage the electric system reliably and economically.

To this end, in this project, we explore and evaluate different combinations of two free and open-source tools, a Numerical Weather Prediction (NWP) system and a PV modeling library. The Weather Research and Forecasting (WRF) Model is a mesoscale NWP system designed for both atmospheric research and operational forecasting applications [33, 34]. pvlib-python is a community-supported tool that provides a set of functions and classes for simulating the performance of photovoltaic energy systems $[35,36]$. pvlib-python was originally ported from the homonymous MATLAB toolbox developed at Sandia National Laboratories and implements many of the models and methods developed at the Labs. The pvlib-python library comes with some functions to retrieve weather forecast data from some particular web services, thus through minimal modifications, it was adapted to read data produced by our installation of the WRF model.

In this work, we present a comparison of the outcomings of two strategies using off-the-shelf configurations of the NWP model to provide radiation input to the PV modeling library. This was intended to set the grounds for future developments, evaluating each approach.

WRF-direct: In this first strategy, we directly use surface radiation direct and diffuse components provided by WRF. These components were affected by the aerosol optical depth (AOD) measured by the SAVER-Net Project [37] at the closest moment [36].

WRF-coverage: In this second case, we post-process WRF output through the NCEP Unified Post Processing System (UPP) [38] obtaining low, mid, and high-level cloud covers. Those cloud coverages are then composed to get a total cloud cover value, and processed through pvlib-python routines to get the surface global radiation [39] which is then decomposed into direct and diffuse components using the DISC model [40].
Figure 11 shows a comparison of the surface global irradiance $(\mathrm{GHI})$ forecasted by each of the two strategies along nine days during December 2017, for a location in Buenos Aires, Argentina. GHI results are compared with measurements provided by the SAVER-Net Project. Minutely measurements were averaged over 12-minute periods to make them coincide with WRF output frequency. This period was selected as it contains days with a clear sky, partial cloudiness and full overcast conditions. NCEP Reanalysis data [41] was used as initial and boundary conditions for WRF. In this setting, WRF is acting as a dynamical interpolator of the reanalysis conditions. Looking at days with clear sky conditions (Dec 18, 20, 24 and 25) reveals that WRF-direct strategy better agrees with measurements than WRF-coverage, exposing the need to take into account the AOD effect. Conversely, days with partial cloudiness (Dec 21 and 23) or overcast (Dec 19) expose a WRF-direct tendency to overestimate radiation, while WRF-coverage gives lower values than, in any case, do not follow measurements much close. There are also days with intermittent cloudiness (Dec 22 and 26) which both strategies fail to detect.

A useful measure to evaluate model behavior is to compare aggregated daily energy. Figure 12 shows the total daily energy predicted by each strategy, and how they compare against measurements. In this case, the tendency is repeated with WRF-direct acting better in clear sky conditions, but always overestimating radiation.

To acquire some insight into models internal behavior, direct normal (DNI) and diffuse (DHI) irradiance components as obtained from both strategies can be analyzed. Results are shown in Figure 13. In both strategies, the diffuse component is increased over the direct component in days with high cloudiness. Strange behavior is observed in clear sky days near noon for the WRF-coverage DNI results, dent-shape limits the maximum value attained. This is compensated by an increase in the $\mathrm{DHI}$ component. This appears to be a spurious effect caused by the DISC model implementation. Comparison against DNI or DHI measurements and a deep analysis of the DISC model will be needed to asses which behavior better represents reality.

After global radiation is decomposed in direct and diffuse components, the pipeline to get generation data from weather prediction models for a particular PV system is as follow (see Figure 14):

1. Any of the two strategies provides a forecast for irradiances: global horizontal (GHI), diffuse horizontal (DHI), and direct normal (DNI); and other variables which affect the PV system working condition like temperature and wind speed as already exposed.

2. Irradiance data is processed through pvlib-python 


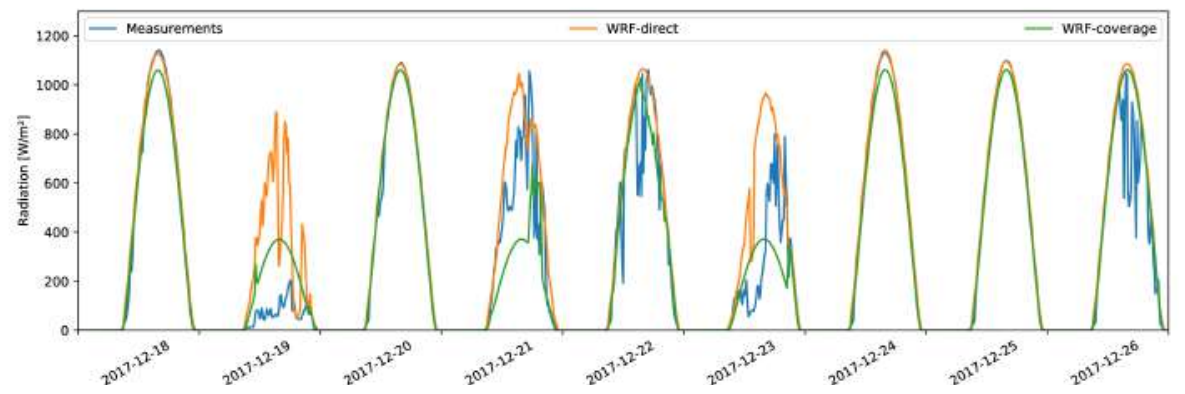

Figure 11 Surface global horizontal irradiance (GHI) variation during a 9-day period in December 2017. Comparison between prediction of the WRF-direct and WRF-coverage strategies and measurements

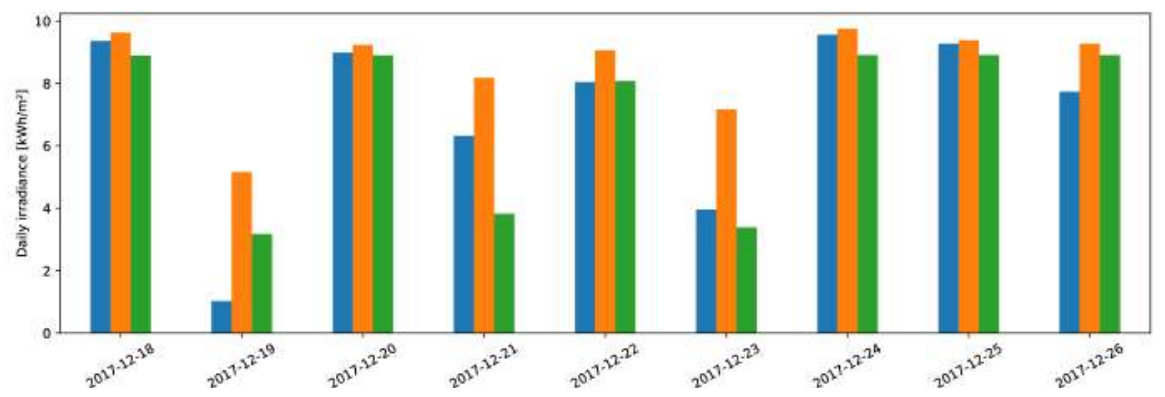

Figure 12 Total daily energy for each of the 9 day in December 2017. Comparison between prediction of the WRF-direct and WRF-coverage strategies and measurements

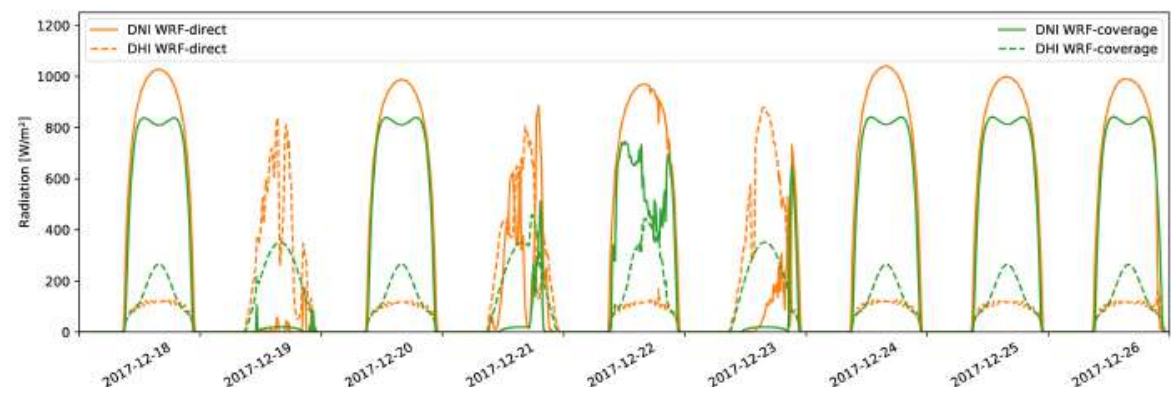

Figure 13 Direct normal (DNI) and diffuse (DHI) variation during a 9-day period in December 2017. Prediction of the WRF-direct and WRF-coverage strategies

to obtain irradiance components projected on the PV modules plane-of-array (POA): global, direct, and diffuse.

3. Considering the configuration (number of series and parallel connected) and model of PV modules, the pvlib-python is used to forecast the DC power production.

4. Regarding the information about the system inverter, the AC power is forecasted.

To evaluate the performance of the above-mentioned pipeline, power generation measurements from a $19.6 \mathrm{~kW}$ PV plant property of the Environmental Protection Agency of Buenos Aires city were used as a comparison (https://www.sunnyportal.com/ Templates/PublicPageOverview . aspx?page= cbf7bf62-4171-430d-ab0d-46854669a126\&plant= e4f89244-d746-42e2-9f72-909d76da9d66). This plant can be regarded as a typical community distributed generation installation. The actual plant is composed of two $5 \mathrm{kWp}$ (the 'p' letter denotes peak power) inverters connected to $250 \mathrm{Wp}$ PV modules and one $8 \mathrm{kWp}$ inverter connected to $215 \mathrm{Wp}$ PV modules. The simulated PV system was defined inside pvlib-python as composed by one inverter (SMA America STP5000TL) and modules (Amerisolar AS-6P30-250W) arranged in two strings of 11 modules each, equivalent to the circuit of one of the $5 \mathrm{kWp}$ inverters of the actual plant. To generalize the results, power generation was normalized concerning peak plant power. The installation 15-minutes normalized power is shown in Figure 15. The outcomes of the forecasting pipeline with inputs provided by the two strategies are 


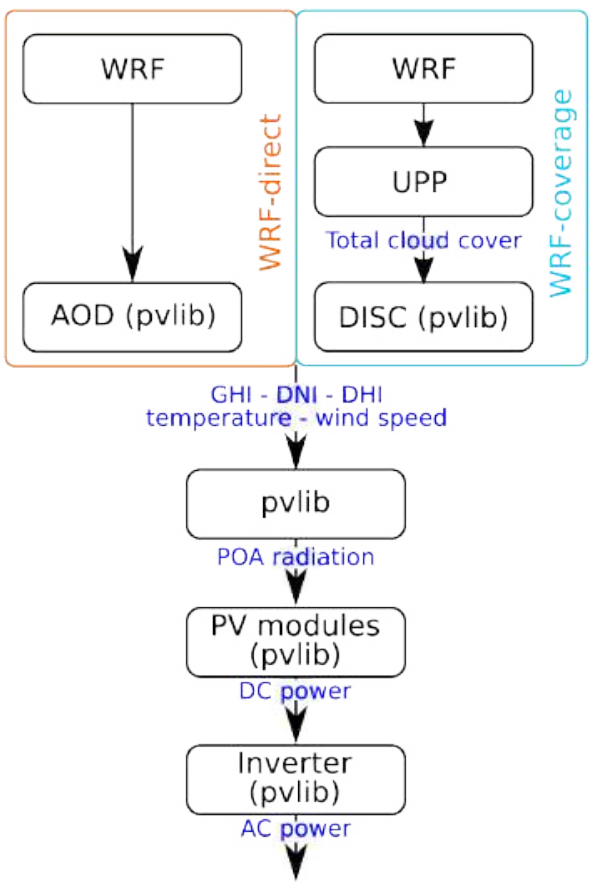

Figure 14 PV generation forecast pipeline including the two meteorological variables forecasting strategies

also shown therein. In this case, an excellent agreement is given for clear sky days with the WRF-direct pipeline, stressing the importance of the AOD correction. In cloudy days, the results resemble the differences observed in $\mathrm{GHI}$.

As a summarizing measure of this section the daily normalized energy forecasted by each strategy pipeline is compared with the actual generation of the plant in Figure 16. It can be observed that both strategies tend to overestimate generation in cloudy days and underestimate generation in clear-sky days, with WRF-direct better forecasting clear-sky days generation with respect to WRF-coverage. In these cases, the error is less than $10 \%$, but on cloudy days it raises considerably.

The two proposed strategies allow forecasting the generation of individual PV systems. Although figures show 12-min data for convenience, more frequent sampling can be obtained as the actual model time step is smaller, allowing to capture the dynamics of the possible generation. The smallest sampling step not producing spurious dynamics should be evaluated. For modeling multiple systems assigned to the same WRF grid point, for which no difference will be detected in the radiation forecast, equivalent systems could be defined. Results suggest that:

- Other WRF and pvlib-python configurations need to be explored, assessing their forecasting performance,

- Some type of cloudiness lintermittent on Dec 22 and
26 , constant in the morning Dec 23) is not detected by WRF-direct in the adopted configuration, more insight needs to be gained to clarify this behavior,

- The effect of cloud coverage on radiation is overestimated by WRF-coverage,

- DISC model misbehaves near noon on clear sky days, other models could be evaluated.

- Performance of PV generation for days with great diffuse components need to be verified against radiation components for days with high cloudiness is verified.

At the moment, development efforts are focused on model calibration and validation. Several configurations and submodel options could be chosen and a systematic error quantification study is underway.

\section{Conclusions and future work}

This article presented an overall description of the CC-SEM project in conjunction with preliminary results. In this project, it is proposed to build an integrated platform for smart monitoring, controlling, and planning energy consumption and generation in urban scenarios. In particular, three main activities are described.

In the axis 1, the defined goal is to automatically manage energy for home devices, our main contribution consists in the development of a low-cost loT device capable of monitoring, operating, and controlling home appliances according to predefined rules. The prototype was successfully used to gather part of the data used in the remainder of the project.

Also, we address an important latency-sensitive metering issue in the context of a Smart Grid: delivering massive simultaneous ORM messages. Our results suggest that NB-IoT is a suitable technology for the platform that is proposed in this work.

We propose to utilize Big Data techniques for analyzing domestic energy consumption and smart planning in the axis 2. The contribution is based on the analysis of domestic consumption patterns to help predicting home consumption through a sequence of previous measurements. The obtained estimations are good enough to guide a recommendation system or to help to detect potential network overloads.

In the last activity, axis 3, the objective was to simulate an electrical network (which includes three stages: generation, transmission, and distribution) with the addition of a communication network. A forecasting and 


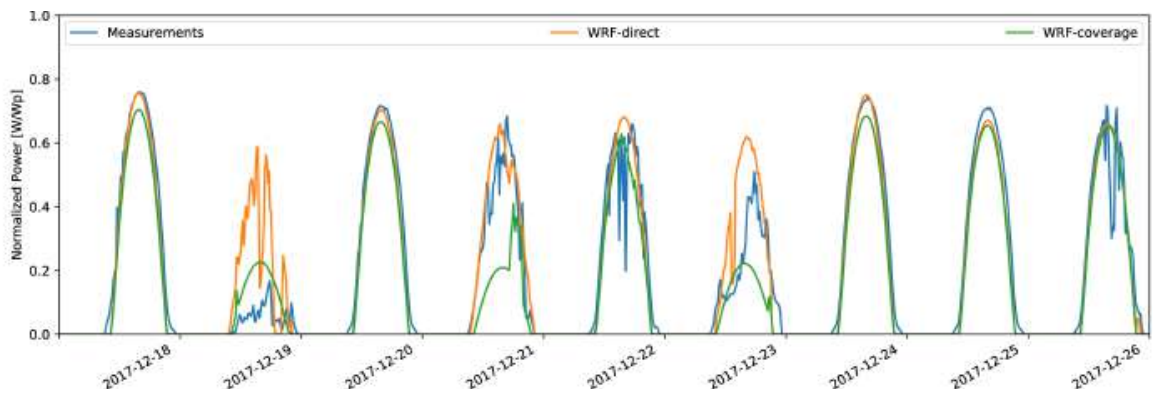

Figure 15 Normalized power generation forecasted by the two strategy pipelines compared with an actual PV plant in Buenos Aires, Argentina. Instant power is compared with plant peak power en each case

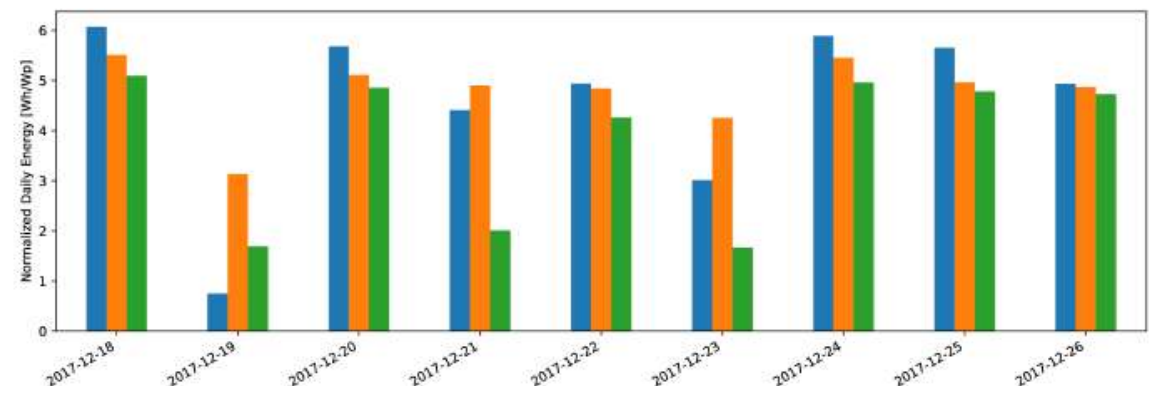

Figure 16 Normalized daily energy generation forecasted by the two strategy pipelines compared with an actual PV plant in Buenos Aires, Argentina, compared with actual plant generation

performance evaluation methodology to forecast the generation of individual PV systems is proposed. An NWP model is combined with a PV modeling library called pvlib-python. This approach results in utter importance due to PV systems representing a major technology for massive renewable energy generation within urban scenarios.

The main lines for current and future work include performing a deep analysis of home consumption patterns to better characterize specific behaviors of citizens regarding other data sources, including socio-economic, weather, and neighborhood-related data. In this regard, in the current stage of the project, we are working on performing more comprehensive experiments with real data provided by National Energy Administration in Uruguay (UTE) applying ANN and other computational intelligence methods. Preliminary results suggest the effectiveness of the approach. The project will also continue to explore loT development with new sensor technology and advance in establishing a program of controlled scaled domestic measurements. Finally, regarding smart grid, new communications standards will be analyzed and their impact on protocols and infrastructure will be analyzed focusing on creating a new communication layer on top of the electric network.

\section{Declaration of competing interest}

None declared under financial, profesional and personal competing interests.

\section{Acknowledgment}

CC-SEM project is supported by the STIC-AmSud regional program (France-South America).

\section{References}

[1] S. A. Roosa, S. Doty, and W. C. Turner, Energy management handbook, 9th ed. The Fairmont Press, Sep. 2018.

[2] A. Towsend, Smart Cities: Big Data, Civic Hackers, and the Quest for a New Utopia, 1st ed. W. W. Norton \& Company, Oct. 2013.

[3] A. Soares, C. Antunes, C. Oliveira, and A. Gomes, "A multi-objective genetic approach to domestic load scheduling in an energy management system," Energy, vol. 77, pp. 144-152, 2014.

[4] A. Zakariazadeh, S. Jadid, and P. Siano, "Economic-environmental energy and reserve scheduling of smart distribution systems: A multiobjective mathematical programming approach," EnergConversManage, vol. 78, pp. 151-164, Feb. 2014.

[5] Y. Wahyuddin, "To what extent the grand lyon metropole can harness the smart meter project towards the governance of territorial climate energy plan (PCET) study case: Smart electric lyon project initiated by EDF [french electric utility company]," in Proc of the International Conference on Public Policy, 2017.

[6] S. Gupta, M. Reynolds, and S. Patel, "Electrisense: Single-point sensing using EMI for electrical event detection and classification in 
the home," in Proc of the 12th ACM Int Conf on Ubiquitous Computing. New York, NY, USA: ACM, 2010, pp. 139-148.

[7] A. Spagnolli, N. Corradi, L. Gamberini, E. Hoggan, G. Jacucci, C. Katzeff, L. Broms, and L. Jonsson, "Eco-feedback on the go: Motivating energy awareness," Computer, vol. 44, no. 5, pp. 38-45, Apr. 2011.

[8] L. Gamberini, A. Spagnolli, N. Corradi, G. Jacucci, G. Tusa, T. Mikkola, L. Zamboni, and E. Hoggan, "Tailoring feedback to users' actions in a persuasive game for household electricity conservation," in Persuasive Technology. Design for Health and Safety, ser. Lecture Notes in Computer Science, M. Bang and E. Ragnemalm, Eds., vol. 7284. Berlin, Germany: Springer, 2012, pp. 100-111.

[9] E. Costanza, S. Ramchurn, and N. Jennings, "Understanding domestic energy consumption through interactive visualization: A field study," in Proc of the 2012 ACM Conf on Ubiquitous Computing. New York, NY, USA: ACM, 2012, pp. 216-225.

[10] C. Chen, S. Duan, T. Cai, B. Liu, and G. Hu, "Smart energy management system for optimal microgrid economic operation," IET Renewable Power Generation, vol. 5, no. 3, pp. 258-267, May 2011.

[11] E. Luján, A. Otero, S. Valenzuela, E. Mocskos, L. A. Steffenel, and S. Nesmachnow, "Cloud computing for smart energy management (CC-SEM project)," in Smart Cities, S. Nesmachnow and L. Hernández Callejo, Eds., vol. 978. Cham: Springer, 2019, pp. 116-131.

[12] J. Rabaey, M. Ammer, J. da Silva, D. Patel, and S. Roundy, "Picoradio supports ad hoc ultra-low power wireless networking," Computer, vol. 33, no. 7, pp. 42-48, 2000.

[13] A. Whitmore, A. Agarwal, and L. Da Xu, "The internet of things-a survey of topics and trends," Inform Syst Front, vol. 17, no. 2, pp. 261-274, Apr. 2015.

[14] E. Orsi and S. Nesmachnow, "lot for smart home energy planning," in XXIII Congreso Argentino de Ciencias de la Computación, 2017.

[15] E. Orsi and S. Nesmachnow, "Smart home energy planning using loT and the cloud," in Proc of the IEEE URUCON. IEEE, Oct. 2017, pp. 1-4.

[16] C. A. Ramírez, R. C. Barragán, G. García-Torales, and V. M. Larios, "Low-power device for wireless sensor network for smart cities," in Proc of the IEEE MTT-S Latin America Microwave Conference (LAMC). IEEE, Dec. 2016, pp. 1-3.

[17] Y. P. E. Wang, X. Lin, A. Adhikary, A. Grovlen, Y. Sui, Y. Blankenship, J. Bergman, and H. S. Razaghi, "A primer on 3gpp narrowband internet of things," IEEE Commun Mag, vol. 55, no. 3, p. 117-123, Mar. 2017.

[18] V. Nair, R. Litjens, and H. Zhang, "Assessment of the suitability of NB-loT technology for ORM in smart grids," in Proc of the European Conf on Networks and Communications (EuCNC). IEEE, Jun. 2018, pp. 418-423.

[19] TSGR, "LTE; E-UTRA; Physical channels and modulation (3GPP TS 36.211 version 14.4.0 Release 14)," ETSI Standard, 2017.

[20] TSGR, "LTE; E-UTRA; Multiplexing and channel coding (3GPP TS 36.212 version 14.4.0 Release 14)," ETSI Standard, 2017.

[21] E. Luján, J. A. Zuloaga Mellino, A. D. Otero, L. Rey Vega, C. G. Galarza, and E. E. Mocskos, "Extreme coverage in $5 \mathrm{~g}$ narrowband iot: a lut-based strategy to optimize shared channels," arXiv e-prints, p. arXiv:1908.02798, Aug. 2019.

[22] J. A. Zuloaga Mellino, E. Luján, A. D. Otero, E. E. Mocskos, L. R. Vega, and C. G. Galarza, "Lite NB-loT Simulator for Uplink Layer," in 2019 XVIII Workshop on Information Processing and Control (RPIC), Sep. 2019, pp. 286-291.

[23] E. Luján and J. A. Z. Mellino, "Lite NB-loT NPUSCH Simulator," https://github.com/CSC-CONICET/ Lite-NBIoT-NPUSCH-Simulator, 2018.
[24] G. Ferrari, P. Medagliani, S. Di Piazza, and M. Martalò, "Wireless sensor networks: Performance analysis in indoor scenarios," EURASIP Journal on Wireless Communications and Networking, vol. 2007, no. 1, p. 081864, Mar 2007.

[25] P. T. et al., "Que no se corte," https://www.dc.uba.ar/ se-realizo-tecnox-una-competencia-cientifica-innovadora/, 2018, last access: December 2, 2019.

[26] W. Kong, Z. Y. Dong, Y. Jia, D. J. Hill, Y. Xu, and Y. Zhang, "Short-term residential load forecasting based on Istm recurrent neural network," IEEE T Smart Grid, vol. 10, no. 1, pp. 841-851, Jan. 2019.

[27] K. Amarasinghe, D. L. Marino, and M. Manic, “Deep neural networks for energy load forecasting," in Proc of the IEEE 26th Int Symp on Industrial Electronics, Jun. 2017, pp. 1483-1488.

[28] D. Dheeru and E. Karra Taniskidou, " $\mathrm{UCl}$ machine learning repository," 2018, last access: October 25, 2018. [Online]. Available: http://archive.ics.uci.edu/ml

[29] S. Hong, "Individual household electric power consumption," 2015, last access: October 25, 2018. [Online]. Available: https: //sunhaehong.wordpress.com/2015/08/07

[30] D. L. Marino, K. Amarasinghe, and M. Manic, "Building energy load forecasting using deep neural networks," in Proc of IECON 2016 42nd Annual Conf of the IEEE Industrial Electronics Society. IEEE, Oct. 2016, pp. 7046-7051.

[31] K. Zhou and S. Yang, "Understanding household energy consumption behavior: The contribution of energy big data analytics," Renew Sust Energ Rev, vol. 56, pp. 810-819, Apr. 2016.

[32] Z. J. Kolter and M. J. Johnson, "Redd: A public data set for energy disaggregation research," in Proc of the 1st KDD Workshop on Data Mining Applications in Sustainability, 2011.

[33] W. Skamarock, J. Klemp, J. Dudhia, D. Gill, D. Barker, M. Duda, X. Huang, W. Wang, and J. Powers, "A Description of the Advanced Research WRF Version 3," National Center for Atmospheric Research, Tech. Note NCAR/TN-475+STR, 2008.

[34] N. C. for Atmospheric Research (NCAR), "Weather Research and Forecasting (WRF) Model," https://www.mmm.ucar.edu/ weather-research-and-forecasting-model, 2018, last access: October 25, 2018. [Online]. Available: https://www.mmm.ucar. edu/weather-research-and-forecasting-model

[35] W. F. Holmgren, R. W. Andrews, A. T. Lorenzo, and J. S. Stein, "PVLIB Python 2015," in Proc of the 2015 IEEE 42nd Photovoltaic Specialist Conference (PVSC). IEEE, Jun. 2015, pp. 1-5.

[36] W. F. Holmgren and D. G. Groenendyk, "An open source solar power forecasting tool using PVLIB-Python," in Proc of the 2016 IEEE 43rd Photovoltaic Specialists Conference. IEEE, Jun. 2016, pp. 0972-0975.

[37] SAVER-Net Project, http://www.savernet-satreps.org, 2018, last access: October 25, 2018.

[38] N. C. for Environmental Prediction (NCEP), “NCEP Unified Post Processing System (UPP)," https://dtcenter.org/upp/users, 2017. last access: October 25, 2018. [Online]. Available: https://dtcenter. org/upp/users/

[39] D. P. Larson, L. Nonnenmacher, and C. F. Coimbra, “Day-ahead forecasting of solar power output from photovoltaic plants in the american southwest," Renew Energ, vol. 91, pp. 11 - 20, 2016.

[40] E. L. Maxwell, "A quasi-physical model for converting hourly global horizontal to direct normal insolation," Solar Energy Research Inst., Golden, CO (USA), Tech. Rep. SERI/TR-215-3087, Aug 1987.

[41] N. C. for Environmental Prediction, "NCEP GDAS/FNL 0.25 Degree Global Tropospheric Analyses and Forecast Grids," https://doi.org/ 10.5065/D65Q4T4Z, Boulder C0, 2015, last access: October 25, 2018. 